\title{
Jordanian Nurses' Knowledge of Preventing Surgical Site Infections in Acute Care Settings
}

\author{
Mahmoud N. Qasem, Issa M. Hweidi \\ Department of Adults Health Nursing, Jordan University of Science and Technology, Irbid, Jordan \\ Email: albedawy1986@yahoo.com
}

How to cite this paper: Qasem, M.N. and Hweidi, I.M. (2017) Jordanian Nurses' Knowledge of Preventing Surgical Site Infections in Acute Care Settings. Open Journal of Nursing, 7, 561-582. https://doi.org/10.4236/ojn.2017.75043

Received: January 6, 2017

Accepted: May 22, 2017

Published: May 25, 2017

Copyright (๑) 2017 by authors and Scientific Research Publishing Inc. This work is licensed under the Creative Commons Attribution International License (CC BY 4.0).

http://creativecommons.org/licenses/by/4.0/ (c) (i) Open Access

\begin{abstract}
Background: Surgical Site Infection (SSI) is one of the most common healthcare-associated infections, its account for up to $16 \%$ of all healthcare-associated infections worldwide. The SSIs can contribute to post-operative morbidity, prolonged recovery, delayed discharge and increasing cost. Nurses' knowledge of the evidence-based recommendations is necessary to provide high-quality nursing care. Aims: To assess the level of Jordanian nurses' knowledge regarding evidence based guidelines for the prevention of SSIs, to describe the relationship between nurses' knowledge and selected sociodemographics, to examine the differences in nurses' knowledge with respect to selected dichotomized variables, and to identify the most significant predictors of Jordanian nurses' knowledge regarding evidence based guidelines for the prevention of SSIs. Design: Cross-sectional design. Sample: Two hundred registered nurses at four targeted hospitals were recruited conveniently. Results: The mean of the total knowledge scores of the sample was 3.28 out of nine $(\mathrm{SD}=1.72$, range $=0-7)$, the median was 3 out of nine. There were a statistically significant correlation between all tested continuous sociodemographics variables and the total knowledge score $(p>0.05)$. There is a significant difference in nurses' knowledge between those who are attending to special surgical related training course and who are not. The most significant predictors of Jordanian nurses' knowledge were: the number of credit hours attended by nurses for surgical-related training courses and the total years of work experience in nursing. These variables explained $16.7 \%$ of variance. Conclusion: The results of this study shed light on the obstacles that hampers the Jordanian nurses' knowledge regarding evidence based guidelines for the prevention of SSIs in acute care settings. However, the successful implementation of infection control measures, particularly SSIs prevention measures, and well-structured continuing education programs are considered as a substantial element that would improve nurses' knowledge.
\end{abstract}




\section{Keywords}

Prevention, Evidence Based Guidelines, Nurses' Knowledge, Infection Control, Surgical Site Infection

\section{Introduction}

Surgical site infection (SSI) is a wound infection that developed postoperatively, it is considered as a complication of the surgical procedure or of the post-operative caring of the surgical site [1]. Surgical site infection is an infection that developed after surgery in the part of the body where the surgery took place. Surgical site infections can sometimes be superficial infections involving the skin only. Other surgical site infections are more serious and can involve tissues below the skin, organs, or implanted material [2] [3].

The SSI is one of the most common healthcare-associated infections, its account for up to $16 \%$ of all healthcare-associated infections worldwide [2]. The SSI, globally, is considered as a challenging problem that was facing all surgical patients who are acutely ill. The annual incidence rate of SSI in general population was $20.3 \%$ in Nigeria [4], $16 \%$ in India [5], and $14.7 \%$ in Japan [6]. The annual incidence rate of SSI in Jordan among Coronary Artery Bypass Graft Surgery (CABG) patients reached up to $16.8 \%$ [7].

Surgical site infections have too many risk factors that may contribute to its incidence that include: 1) all operations that involve the abdomen; 2) all operations that last longer than two hours; 3 ) contaminated or dirty site of wound; and, 4) patients who had three or more co-morbidities when they left hospital [8]. In addition to contaminated or dirty site of wound, high body mass index, re-operation and use of post-surgical drain increased the infection risk of surgical site infection among lumpectomies and mastectomies patients [9].

Furthermore, there are other contributing risk factors among colorectal cancer patients, such as: disseminated cancer, ileostomy, patient temperature less than $36^{\circ} \mathrm{C}$ for more than 60 minutes, and high blood glucose level [10]. There are Pre-operative influences on surgical site infection incidence such as: length of hospital stay, cigarette smoking, miss usage of antibiotic prophylaxis, bowel preparation, hair removal and patients shower use [8]. These factors are modifiable by minimizing patient's length of pre and post-operative stay, decline patient's cigarette smoking, giving appropriate systemic prophylactic antibiotic, proper bowel preparation, remove hair with clippers with a disposable head as close to the time of surgery as possible, and showering with ordinary soap and water the night before surgery [8].

Healthcare-associated infections are still considered as a major challenging problem clinically. In United States, its cost may reach up to $\$ 45$ billion yearly. SSI is one of the most common causes of post-operative morbidity [11]; such infections contribute to prolonged recovery, delayed discharge and increase costs to both patients and health service [12]. The SSI could prolonged surgical pa- 
tients' stay approximately 14 days more and increase the direct cost burden $10.232 \$$ per patient [13]. Furthermore, the SSI indirect cost can reach up to $97.433 \$$ per patient [13].

There are major effects of SSI on postoperative hospitalization stay, where infected surgical site could extent patient's hospitalization stay up to 16 days more [14]. Furthermore, it's raising the hospitalization cost more than 100\% [14].

Nurses at all levels of work have a crucial role in controlling and preventing infection [15]. Knowledge of the related evidence-based guidelines regarding the prevention of SSIs is necessary to provide high-quality nursing care [16].

Nurses should apply standards of infection control and precautions to prevent cross contamination from both recognized and unrecognized sources of infection, and transmission-based precautions for special indicated cases [17]. In patients who have surgical procedures such as; caesarean section, hip arthroplasty, knee arthroplasty, reduction of long bone fracture, or repair of neck of femur, nurses should collaborate with other health team members to control and prevent surgical site infection occurrence [2]. Nurses should continually learn the updates of evidence-based in prevention of surgical site infections [17]. Nurses play a key role in wound management and their theoretical understanding of basic wound management expected to influence the quality of wound therapy fundamentally [18]. Subsequently, education of health care professionals can improve their knowledge level, thus promoting infection prevention guidelines implementation which directly contributes to health care associated infections reduction [19]. In a recent qualitative study that compared knowledge and attitudes towards Evidence-Based Nursing Practice (EBNP) among nurses depending on their occupational positions. The level of knowledge about EBNP in both groups departmental nurse, and charge nurse was inadequate and needs urgent supplementation of knowledge and skills of nurses with reference to this subject area [20]. However, a gap exists between the best evidence and practice with regards to SSI prevention. Awareness of evidence is the first step in knowledge translation [15].

In Belgium, a study, conducted to evaluate nurses' knowledge regarding evidence based guidelines for the prevention of SSIs in order to identify their specific educational needs, male nurses' were found to be more knowledgeable than their female colleagues concerning the implementation of the SSIs prevention guidelines [16]. In Australia, another study found that there was a positive relationship between the number of years of work experience and nurses' level of knowledge regarding SSIs prevention [21].

\subsection{Aims of the Study}

1) To assess the level of Jordanian nurses' knowledge regarding evidence based guidelines for the prevention of SSIs.

2) To describe the relationship between nurses' knowledge regarding SSI prevention and selected personal sociodemographics that were measured on both continuous and dichotomous variables. 
3) To examine the differences in Jordanian nurses' knowledge regarding SSI prevention with respect to gender, marital status, and level of education.

4) To identify the most significant predictors of Jordanian nurses' knowledge regarding SSI prevention.

\subsection{Research Questions}

1) What is the level of Jordanian nurses' knowledge regarding evidence based guidelines for the prevention of SSIs in acute care settings?

2) Is there any association between nurses' knowledge regarding evidence based guidelines for the prevention of SSIs in acute care settings and selected sociodemographics measured as continuous variables?

3) Are there any significant differences in Jordanian nurses' knowledge regarding evidence based guidelines for the prevention of SSIs in selected sociodemographics measured as dichotomized variables?

4) What are the most significant predictors of Jordanian nurses' knowledge regarding evidence based guidelines for the prevention of SSIs in acute care settings?

\section{Literature Review}

\subsection{Nurses' Knowledge Regarding Evidence Based Guidelines for the Prevention of SSIs}

Nurses have a golden role in preventing surgical site infection occurrence [15]. The higher nurses' level of knowledge, the fewer incidence of surgical site infection [16]. Nurses require to be knowledgeable regarding the cause, effect and management of SSI to ensure optimum patient's outcomes following surgery [2].

What the nurse should know regarding surgical site infection prevention? Nurses should be aware of surgical site infection definitions, classifications, risk factors and the population at risk, signs and symptoms of surgical site infection, the antibiotic prophylactic use, pre-operative skin preparations, post-operative surgical site caring, infection control standards and surgical site infection prevention strategies, and to advocate their clients at all situations [8].

\subsection{Nurses' Knowledge and Surgical Site Infection Classifications}

It is important for nurses to distinguish between surgical site infection classes. Which are superficial incisional, deep incisional, and organ/space SSIs, depending upon the tissue or body part involved [2].

In a descriptive study, that evaluated Intensive Care Units (ICU) nurses' knowledge of surgical site infection prevention guidelines using a newly developed tool that used in this study, only $7 \%$ of nurses knew the correct classification of surgical site infection, while $46 \%$ knew that stitch abscesses are not to be reported as SSI. Furthermore, only $2 \%$ recognized the exact time frame in which emerging superficial incisional infections are classified as SSIs. Eventually, it was reported that overall scores of nurses' knowledge regarding evidence based guidelines for the prevention of SSIs were poor; thereby, it may reflect nurses' 
daily practice [16]. However, the study's target population were all nurses who work in ICU, regardless to the ICU specialty, so, the data may be collected form medical ICU nurses who were not exposed to surgical experience which may negatively impact nurses' knowledge scores.

\subsection{Nurses' Knowledge and Surgical Site Infections: Signs and Symptoms}

Usually, following surgery, patients experience; pain, swelling and redness around the wound as part of the normal wound healing process [22]. However, these symptoms may intense when SSI occur [3]. Furthermore, SSIs are associated with redness, heat, pain, swelling, hyperthermia as temperature greater than 38 degrees centigrade, purulent discharge, and abscess or cellulitis directly related to the surgical site [23]. Moreover, in Sweden, a study conducted to evaluate quality of life of patients with sternal wound infection after cardiac surgery, it was found that if those patients survive, SSI is a very serious complication that negatively impact their quality of life [24]. Another study, that investigated the long term effect of SSI on several quality of life measures in patients undergoing knee and hip surgery, it was found that SSI significantly affected patients' mobility, their independency of living, and their psychological health [25].

\subsection{Nurses' Knowledge and Surgical Site Infections Risk Factors}

Several factors increase the risk of developing SSI. These factors are divided into modifiable and non-modifiable factors, thus, nurses' awareness of these factors especially those that are modifiable can help in SSI prevention implementation strategies [2]. Nurses should be aware of the extrinsic factors such as: pre-operative skin preparation, antibiotic prophylaxis use, and post-operative surgical site caring; which can be manipulated to reduce the risk of SSI [8].

Pre-operative skin preparation such as skin disinfection and hair removal are playing an important role in preventing SSI [8]. In an experimental study, that conducted in Turkey to determine the effect of preoperative skin preparation procedures performed by nurses on postoperative surgical site infection in abdominal surgery; the study participants were divided into two groups (control group, $n=39$ ) and (interventional group, $n=43$ ), control group participant's skin were mostly prepared by shaving with a razor blade. In contrast, clippers were used to prepare $55.8 \%$ of interventional group participant's skin, while $44.2 \%$ of them were not treated with the clipper because their wounds were cleaned. All interventional group participants had a chlorhexidine bath at least twice after being hospitalized and at least once a night before the operation under controlled conditions. The difference between control group and interventional group with respect to surgical site infections was statistically significant; which means that preparing skin using clipper at the night before an operation and a $50 \mathrm{ml}$ chlorhexidine bath excluding head area taken twice in the preoperative period were useful measures to reduce SSI during postoperative period [26]. In spite of its powerful design, the small sample size was the major limita- 
tion of this study, which may affect its clinical validity, and generalizability of the results.

In a recent literature review paper that was evaluating the evidence in the literature to identify the best antiseptic agent in terms of skin preparation, it was suggested that cholorhexdine with alcohol is the most effective antiseptic agent in terms of reducing SSI [27].

\subsection{Nurses' Knowledge and Antibiotic Prophylactic Use}

Antibiotic prophylaxis use is one of the measures used in prevention of the development of surgical site infections [15]. Although systemic antibiotics reduce the incidence of SSI significantly [28], the use of antibiotic prophylaxis for prevention of surgical site infection was not effective post breast cancer surgery [29], and not necessary for elective laparoscopic cholecystectomy surgeries [30]. However, the use of antibiotics during the preoperative period for the prevention of surgical site infection remains controversial.

Moreover, accurate and timely administration of antibiotics is a crucial element of pre-operative patient care. Educated nurses can contribute to safe antibiotic administration by preventing medication errors such as; omitted doses, duplicate doses, and incorrect doses. Which indirectly affect post-operative surgical site infections occurrence [31].

\subsection{Nurses' Knowledge: Pre-Operative and Post-Operative Surgical Site Caring}

Post-operative surgical site caring is an important mission in preventing surgical site infection and wound healing. Nurses should know the chain of infection and the modes of infection spread [2].

It is important to know that any break in the skin can help microbial pathogens to get a portal of entry, and hence places the patient at an increased risk of infection, however, postoperative dressings playing a key part alongside universal precautions such as; hand hygiene and aseptic technique [32]. Nurses should be aware that theatre dressing should remain in situ for at least 48 hours before removal, to prevent the entry of microorganisms and promote healing [2]. In a descriptive study, that evaluated ICU nurses' knowledge of the surgical site infection prevention guidelines, $45 \%$ of nurses knew that primarily closed incisions must be protected for 24 to 48 hours [16].

In a recent study, that conducted in Philippines to determine the knowledge and extent of practice of sterile technique among operating room nurses, and to investigate whether certain nurses' characteristics are correlated with their knowledge. The study found that age, gender, length of clinical experience, and numbers of trainings attended are not determinants of the knowledge on the principles of sterile technique [33]. In contrast, an Australian study found a positive relationship between the number of years of work experience and self-reported level of knowledge, as higher scores indicate greater knowledge [21]. Moreover, a Belgium study found that male nurses' were more knowledgeable 
than their female counterparts [16].

\section{Methodology}

\subsection{Design}

A cross-sectional design used to conduct this study.

\subsection{Population and Sampling}

A convenience sample of 200 nurses working at acute care settings from four targeted hospitals was included. The sample size $(\mathrm{n}=200)$ was calculated through a power analysis (Cohen, 1992), which considered the power of $80 \%$, an alpha error of 0.05 , with a confidence interval of $95 \%$, and a large effect size as revealed in the reviewed literature [34]. The population of interest for this study were all eligible registered nurses working in acute care settings; Surgical Intensive Care Unit (SICU), Medical Intensive Care Unit (MICI), Intermediate Care Unit (IMU), Coronary Care Unit (CCU), Coronary Intensive Care Unit (CICU), Surgical wards, and Orthopedic wards in four hospitals: three governmental hospitals, and one university-affiliated teaching hospital in Jordan. These hospitals selected based on: their location and importance. These hospitals are considered as the largest referral hospitals that serves the center and north of Jordan.

Study participants were selected based on a specific eligibility criteria, inclusion criteria for participation in this study include:

1) Age of 20 years and older.

2) Educational level should be BSc or higher.

3) Can read and understand English language efficiently.

4) Work as a full time registered nurse in acute care departments.

5) Jordanian nationality.

\subsection{Instruments}

Two instruments were employed to collect the data for the purpose of this study: the demographic datasheet, it has been developed to collect specific demographic characteristic data including respondent's age, gender, marital status, total years of work experience, nationality, level of education, special surgical related training courses, and monthly income. A multiple choice knowledge test questionnaire that was newly developed by Labeau et al. (2010) based on the Centers for Disease Control and Prevention [1], and SSI prevention guideline [15]. The multiple choice knowledge test questionnaire consisted of nine questions follows the pattern of multiple choice questions, where each question followed by four response alternatives: one answer is correct, two distractors, and the sentence "I do not know", to discourage guessing [16].

Ranks of nurses' knowledge primarily based on the quartile (Q) "Ntile" ranking system which divide the participants in to four equal groups based on their total knowledge percentile as per to the following classification: Q1: cases below 
the 25th percentile, Q2: cases between the 25th and below 50th percentile, Q3: cases between the 50th and 75th percentile, and Q4: cases above the 75th percentile. The main questionnaire consisted of ten questions that was presented to seven experts to assess face and content validity. All experts had at least a master's degree in nursing or medical social sciences, and were, at least locally, involved in research on ICU acquired infections with special interest in SSI. In order to achieve face validity, experts were asked if all questions were clearly worded and would not be misinterpreted. For content validity, the experts evaluated the nursing relevance of the 10 items by using a scale of 1 to 3 , where $1=$ not relevant, 2 = relevant but not necessary, and $3=$ absolutely necessary. Content Validity Index (CVI) was calculated per item, which reflects the proportion of consulted experts agreeing on the content validity of an item. Content validity was beyond the 0.05 level of significance. One question concerned the issue of preoperative bowel preparation, which was identified by the experts as overruled by more recent evidence. This question was deleted from the questionnaire and minor revisions of the wording of some other questions were performed. The experts considered all nine remaining items of the questionnaire relevant for nursing practice. Calculation of the unreported CVI reflected their unanimous agreement with the questionnaire's content and clarity. Cronbach's alpha for the multiple choice Nurses' knowledge test questionnaire was .81 suggesting reasonable level of internal consistency reliability. This result of reliability is comparable to established and unreported high internal consistency of the questionnaire as indicated by the original authors [16].

\subsection{Data Collection Procedure}

The data were collected by the investigator from the four targeted hospitals. Data collected from the last week of September to the end of October 2015, these hospitals were visited randomly at the accessible times during the morning, noon, or night shifts to select the accessible and eligible participants conveniently. Nurses who met the inclusion criteria were asked to participate voluntarily and anonymously by signing the consent form and filling the questionnaire. However, respondent given approximately (ten to fifteen minutes) to fill the questionnaire in nursing station and hand it over manually to the investigator at the same visit time.

\subsection{Statistical Analysis}

Data entered and analyzed statistically using Statistical Package for Social Science (SPSS) version 19. A descriptive statistical analysis was conducted to describe the characteristics of the sample, and their knowledge level. Bivariate spearman's correlations were employed to describe the correlation between continuous selected personal characteristics and knowledge level. The correlation was considered significant at the 0.05 level. A non-parametric Mann-Whitney U-test was employed to detect differences between the medians of knowledge scores in dichotomized selected nurses' characteristics, two-tailed $(P>0.05)$ was 
considered statistically significant. Linear stepwise regression analysis was employed to determine the most significant predictors from all personal characteristics.

\subsection{Ethical Considerations}

Jordan University of Science and Technology Institutional Review Board (IRB) was obtained, and all administrative approvals were obtained from the participant institutions. Thereafter, the permission to use the instrument was obtained from the instrument developer and her colleagues. All participants' rights were ensured based on ethical principles of respect for human dignity, privacy, confidentiality, and autonomy. Participation in the study was voluntarily and anonymously. In addition, each nurse informed regarding his/her right of withdrawal at any time. All participants were informed regarding the purpose of conducting such study. The study procedure explained for each participant, and written consent was signed before filling of the questionnaire to ensure voluntarily participation.

\section{Results}

\subsection{Characteristics of the Sample}

In the four targeted hospitals there were approximately a five hundred and twenty Jordanian registered nurses working in acute care settings. This study recruited two hundred Jordanian registered nurses who were conveniently selected to answer the questionnaire in the targeted hospitals. Nurses' mean age was $27.52+2.92$ years with a range of $23-38$ years. One hundred thirty-six $(68 \%)$ of the sample were male nurses, and (32\%) were female nurses, In terms of their marital status, the data analysis revealed that $(54 \%)$ of the participants were married and (46\%) were unmarried (Table 1).

The mean of the total years of work experience in nursing for the selected sample was $5.3+2.94$ years with a range of $1-15$ years. The mean of total years of work experience in surgical care units was $3.3+2.43$ years with a range of 0.0 - 13 years. Data also revealed that (92\%) of the sample were bachelor-prepared nurses and $(8 \%)$ were master-prepared.

Twenty five percent of the sample underwent a special surgical-related training course and $75 \%$ did not. The total number of credit hours spent by all participants in the training courses was 1782 hours with a mean of $8.91+21.83$ hours (range $=0.0$ to 128). Monthly income of the total sample ranged from 333 JD to $800 \mathrm{JD}(1 \mathrm{JD}=1.41 \$)$ with a mean of $496.22+82.24 \mathrm{JD}$ (Table 1$)$.

\subsection{Jordanian Nurses' Knowledge Level}

Two hundred questionnaires were completed. Data analysis revealed the mean of total knowledge scores of the sample $=3.28(\mathrm{SD}=1.72$, range $=0-7)$, and $(M d n=3)$. Median was used to describe the center of the data because of the data were not normally distributed. The results showed that the overall Jordanian nurses' knowledge level regarding evidence based guidelines for the pre- 
Table 1. Sample characteristics.

\begin{tabular}{|c|c|c|c|c|c|}
\hline Variable & Range & Mean & SD & $\mathbf{N}$ & $\%$ \\
\hline Age (years) & $23-38$ & 27.52 & 2.92 & & \\
\hline \multicolumn{6}{|l|}{ Gender } \\
\hline Male & & & & 136 & 68 \\
\hline Female & & & & 64 & 32 \\
\hline \multicolumn{6}{|l|}{ Marital status } \\
\hline Married & & & & 108 & 54 \\
\hline Unmarried & & & & 92 & 46 \\
\hline Total years of work experience in nursing & $1-15$ & 5.3 & 2.94 & & \\
\hline Total years of work experience in surgical care units & $0-13$ & 3.3 & 2.43 & & \\
\hline \multicolumn{6}{|l|}{ Level of education } \\
\hline $\mathrm{BSc}$ & & & & 184 & 92 \\
\hline MSN & & & & 16 & 8 \\
\hline \multicolumn{6}{|l|}{ Special surgical related training courses } \\
\hline Yes & & & & 50 & 25 \\
\hline No & & & & 150 & 75 \\
\hline Credit hours spent by nurses in the training courses & $0-128$ & 8.91 & 21.83 & & \\
\hline Monthly income (JD) & $333-800$ & 496.22 & 82.24 & & \\
\hline
\end{tabular}

vention of SSIs was low $(\mathrm{Mdn}=3)$. In order to categorize the participants based on their scores we used quartile. Furthermore, the data analysis showed that more than thirteen percent $(n=27)$ of participants were located in Q1 which represent the cases below the 25th percentile, those participants were classified to have very low level of knowledge, while more than forty five percent $(n=91)$ of participants were located in Q2 which represent the 25th to below 50th percentile, those participants were classified to have low level of knowledge. Sixteen percent $(\mathrm{n}=32)$ of participants scores were located in Q3 which represent the 50th to 75th percentile, those participants were classified to have moderate level of knowledge. Furthermore, twenty five percent $(n=50)$ of participants were located in Q4 which represent the cases above the 75th percentile, those participants were classified to have high level of knowledge (Table 2).

Fifty three percent of study participants $(n=106)$ knew that primarily closed incisions must be protected for 24 to 48 hours, and 25.5\% ( $\mathrm{n}=51)$ of the participants were aware that the appropriate time to shower or bathe with uncovered incisions is unresolved. Twenty seven percent $(n=54)$ of the participants knew that postoperative surveillance by itself succeeds in reducing the incidence of SSI, and $69.5 \%(n=139)$ of the participants answered the question number four "Elective surgery on patients with remote site infections should be postponed until the infection has resolved" correctly. The correct classification of SSI was known by $36 \%(n=72)$ of the sample population, while $25 \%(n=50)$ of the study participants were answered question number six "stitch abscesses (mi- 
Table 2. Description of nurses' knowledge level according to their quartile rank.

\begin{tabular}{cccc}
\hline Knowledge level & N & $\%$ & Quartile \\
\hline Very low & 27 & 13.5 & Q1 \\
Low & 91 & 45.5 & Q2 \\
Moderate & 32 & 16 & Q3 \\
High & 50 & 25 & Q4 \\
\hline
\end{tabular}

nimal inflammation and discharge confined to the points of suture penetration) are classified as SSI" as false which was the correct answer. Only 7.5\% $(\mathrm{n}=15)$ of the participants recognized the exact time frame in which emerging superficial incisional infections are classified as SSI. Thirty sex percent $(n=72)$ of the participants knew that preoperative hair removal should take place immediately before surgery, and $49 \%(n=98)$ of the participants knew that electric clippers are recommended to remove the patient's hair at or around the incision site (Table 3).

\subsection{The Correlation between Nurses' Knowledge of SSI Prevention and Selected Nurses' Characteristics}

To describe the correlation between selected continuous variables (personal characteristics) and knowledge level, spearman's correlations were used. Results of correlations analysis indicated that there were a statistically significant correlations $P$-value $<0.01$ between all tested continuous personal characteristics and the total knowledge score; age, total years of work experience in nursing, total years of work experience in surgical care unit, number of credit hours spent in surgical training courses, and monthly income (Table 4).

\subsection{Differences in Jordanian Nurses' Knowledge in Terms of Selected Sociodemographics}

The data were not normally distributed, therefore, a non-parametric MannWhitney U-test was employed to detect differences between medians in Jordanian nurses' knowledge of SSI prevention with respect to gender, marital status, level of education, and attending to special surgical related training course. Data analysis showed that there is a significant difference in nurses' knowledge regarding evidence based guidelines for the prevention of SSIs between those who are attending to special surgical related training course and who are not. Furthermore, there were no statistical significant differences detected in nurses' knowledge regarding evidence based guidelines for the prevention of SSIs with respect to gender, marital status, and level of education subgroups (Table 5).

\subsection{Most Significant Predictors of Jordanian Nurses' Knowledge}

The stepwise regression analysis was employed to determine the most significant predictors from age, gender, marital status, total years of work experience in nursing, total years of work experience in surgical care unit, level of education, number of surgical courses' accredited hours, and monthly income in predicting 
Table 3. Description of nurses' knowledge level with respect to each question.

\begin{tabular}{|c|c|c|c|c|}
\hline \multirow{2}{*}{ Question } & \multicolumn{2}{|c|}{ Correct } & \multicolumn{2}{|c|}{ Incorrect } \\
\hline & $\mathbf{N}$ & $\%$ & $\mathbf{N}$ & $\%$ \\
\hline 1. It is recommended to protect a primarily closed incision ... & 106 & 53 & 94 & 47 \\
\hline $\begin{array}{l}\text { 2. The appropriate time to shower or bathe } \\
\text { ith an uncovered incision is... }\end{array}$ & 51 & 25.5 & 149 & 74.5 \\
\hline 3. Surveillance succeeds in reducing the incidence of SSI. & 54 & 27 & 146 & 73 \\
\hline $\begin{array}{l}\text { 4. Elective surgery on patients with remote site infections } \\
\text { should be postponed until the infection has resolved. }\end{array}$ & 139 & 69.5 & 61 & 30.5 \\
\hline 5. SSIs are classified as ... & 72 & 36 & 128 & 64 \\
\hline $\begin{array}{l}\text { 6. Stitch abscesses (minimal inflammation and discharge } \\
\text { confined to the points of suture penetration) are classified as SSI. }\end{array}$ & 50 & 25 & 150 & 75 \\
\hline $\begin{array}{l}\text { 7. To be classified as SSI, a superficial incisional infection } \\
\text { needs to occur ... }\end{array}$ & 15 & 7.5 & 185 & 92.5 \\
\hline $\begin{array}{l}\text { 8. If the patient's hair at or around the incision site interferes } \\
\text { with the operation, it is recommended to remove it by ... }\end{array}$ & 98 & 49 & 102 & 51 \\
\hline $\begin{array}{l}\text { 9. The recommended time of pre-operative hair } \\
\text { removal in elective surgery is ... }\end{array}$ & 72 & 36 & 128 & 64 \\
\hline
\end{tabular}

Table 4. Spearman's correlation matrix between all continuous variables.

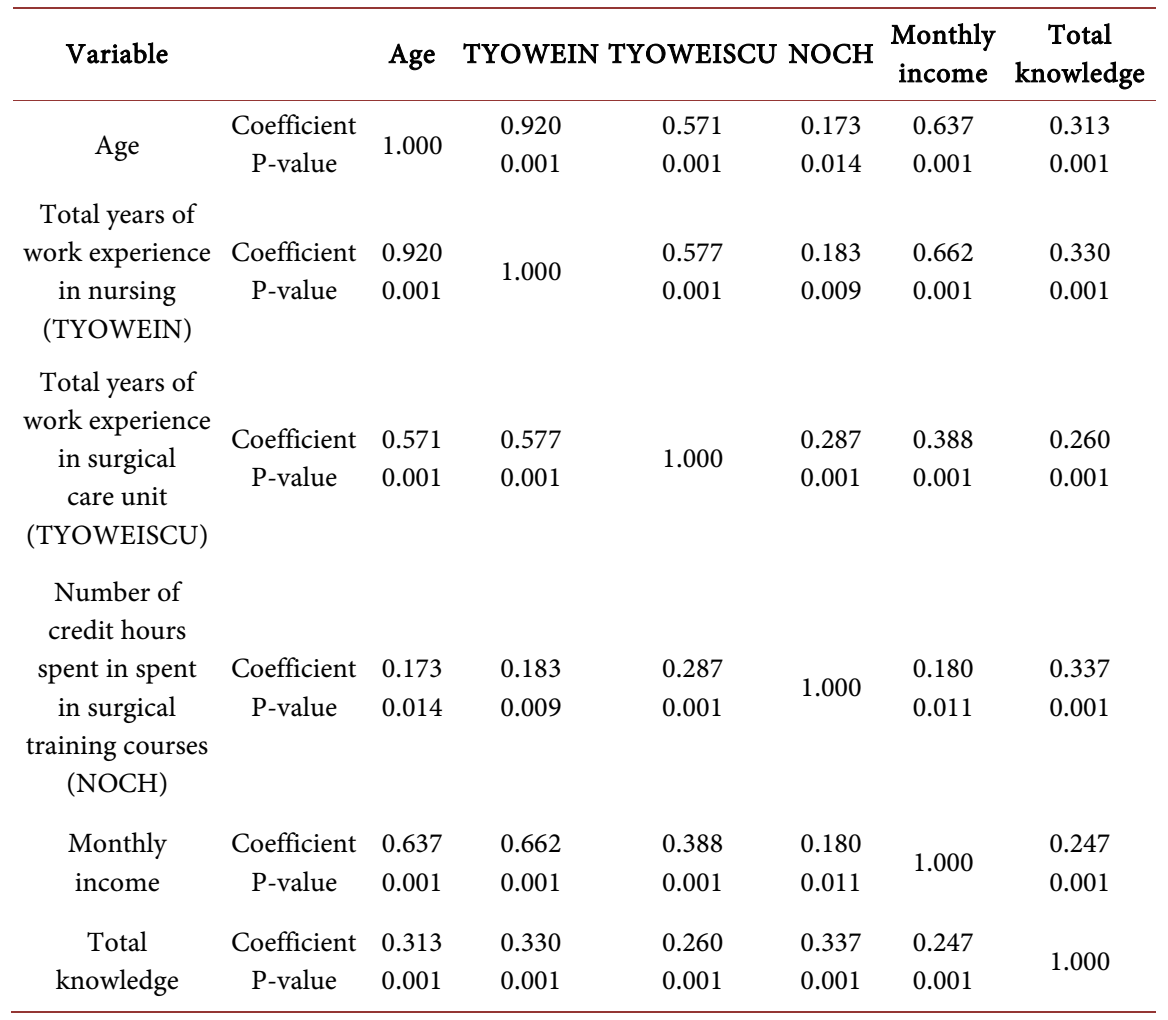

Correlation is significant at 0.01 level (2-tailed).

total knowledge regarding evidence based guidelines for the prevention of SSIs. Two variables; number of credit hours attended by nurses for surgical-related training courses and total years of work experience in nursing were statistically significant predictors of the total knowledge (Table 6). These variables explained 
Table 5. Differences in nurses' knowledge with respect to selected sociodemographics.

\begin{tabular}{ccc}
\hline Variable & N & $P$-value \\
\hline Marital status & 92 & 0.053 \\
Single & 108 & \\
Married & & \\
Gender & 136 & 0.318 \\
Male & 64 & \\
Female & & 0.445 \\
Level of education & 184 & \\
BSc & 16 & \\
MSN & & 0.001 \\
Yes & 50 & \\
No & 150 & \\
\hline
\end{tabular}

Table 6. Significant predictors of nurses' knowledge.

\begin{tabular}{cccc}
\hline Variable & B & Adjusted R square & $\boldsymbol{P}$-value \\
\hline $\begin{array}{c}\text { Number of credit hours attended by nurses } \\
\text { for surgical-related training courses }\end{array}$ & 0.337 & 0.109 & 0.001 \\
$\begin{array}{c}\text { Total years of work experience in nursing } \\
\text { n }\end{array}$ & 0.250 & 0.167 & 0.001 \\
\hline
\end{tabular}

$16.7 \%$ of variance in nurses' knowledge regarding evidence based guidelines for the prevention of SSIs. This model was statistically significant at $(\mathrm{F}=20.94, \mathrm{df}=$ $2, P<0.01)$.

\section{Discussion}

The main purposes of this study were; to assess the level of Jordanian nurses' knowledge regarding evidence based guidelines for the prevention of SSIs, to examine whether certain nurses' characteristics are correlated with their knowledge regarding evidence based guidelines for the prevention of SSIs, to evaluate the differences in Jordanian nurses' knowledge regarding evidence based guidelines for the prevention of SSIs in terms of gender, marital status, and level of education subgroups, and to determine the most significant predictors of Jordanian nurses' knowledge regarding evidence based guidelines for the prevention of SSIs.

The sample was selected conveniently to answer the questionnaire in the four targeted hospitals, which were located in the center and north of Jordan to ensure relative generalizability. The participants who met the inclusion criteria were asked to participate voluntarily in the study by signing the written consent form and answer nine multiple choice questions; where each question have four choices one of these choices was the only correct choice.

The analysis of total knowledge scores of the total sample showed that Jorda- 
nian registered nurses working in acute care settings knowledge level regarding evidence based guidelines for the prevention of SSIs was low based on their median score $(\mathrm{Mdn}=3 ; \%=33.3 \%)$. The sample was not normally distributed (Skewness $=0.262$, Kurtosis $=-0.586$ ), so that, the median is a better representative of central tendency parameter compared to the sample mean score which assumes normality of the data $(\mathrm{M}=3.28, \mathrm{SD}=1.72$, range $=0-7)$. However, $(\mathrm{Mdn}=3)$.

This low level of knowledge regarding evidence based guidelines for the prevention of SSIs is reflecting a serious problem that need immediate intervention in order to improve nurses' knowledge. This lack of knowledge is definitely considered as a main barrier to implement the standard of care in controlling SSIs which is negatively impact the quality of care delivered to the surgical patients, and patient's safety. This claim was congruent with [16], she believed that the poor level of nurses' knowledge regarding evidence based guidelines for the prevention of SSIs considered as a significant obstacle to complying with guidelines for the prevention of SSIs, which is risky on patient's safety and quality of care. However, in a recent study that was conducted to evaluate nurses' knowledge of guidelines for preventing infections associated with peripheral venous catheters, it found that nurses' knowledge was frequently low. It claimed that this low level of knowledge could be a potential risk factor for patient's safety [35].

The result of the total knowledge scores is congruent with other research findings which used the same tool that used in this study to assess nurses' knowledge regarding evidence based guidelines for the prevention of SSIs, it was reported that overall knowledge scores were poor (Labeau et al., 2010), this result attributed primarily to nurses' misconceptions concerning the correct measures to prevent SSIs, and to controversies and contradictions between published recommendations regarding SSIs prevention. However, this result supported also by another research findings investigated nurses' knowledge regarding SSIs prevention which was describe the overall knowledge scores as inadequate [36], their results attributed primarily to lack of exposure to special training courses regarding to preoperative and postoperative nursing interventions in order to prevent SSI.

In this study, the scores that were shifted the data toward low level of knowledge could be attributed to many factors, the main important factors noticed were: firstly, the lack of exposure to special training courses regarding surgical site infection prevention, where a seventy five percent $(n=150)$ of study participants did not attend special training courses regarding surgical site infection prevention. This result was congruent with [36], who reported that only $6 \%$ of three hundred and thirty three nurses who work in surgical wards and operating theatre have had additional exposure to special training courses about surgical site infection prevention where the rest of the study sample (94\%) did not. Tentatively, the lack of exposure to special training courses is a challenging problem facing Jordanian nurses nowadays, which may be due to the lack of conducting well-structured special courses regarding evidence based guidelines for the pre- 
vention of SSIs in Jordanian public governmental hospitals despite of the availability of continuing education units in all of these hospitals, this problem may be refer to defect in continuing education programs implementation which explained by the lack of proper assessment of their nurses educational needs, and the lack of motivation from both the nurses themselves and the hospital administration. However, previous claim was supported by a Jordanian study conducted in 1999 to assess the nurses continuing educational needs. It was concluded that almost two thirds of study participants were not attended continuing education programs in the last previous year. In that study, investigator attributes his result mainly to the lack of motivation to participate in continuing education programs [37].

Nurses' misconceptions concerning the correct measures to prevent SSI was the second main factor that contributed to deviation of the total knowledge score regarding evidence based guidelines for the prevention of SSIs; twenty seven percent $(n=54)$ of study participants correctly recognized that surveillance as a concept succeeds in reducing the incidence of SSI without supplementary preventive measures, while seventy three percent $(n=146)$ did not. This result was congruent with Labeau et al. (2010) who reported that more than half of study participants 54\% were either thought that stitch abscesses are classified as SSI, which is incorrect, or they did not know the concept of stitch abscess. However, this misconceptions may be come from the improper assessment of nurses' educational and learning needs, the lack of highlighting SSI prevention topic as a priority in continuing educational programs, and the lack of available research sources for nurses in order to update and implement evidence based practice. In a recent study that conducted in Jordan to explore the predictors that facilitate or limit research utilization among Jordanian registered nurses, it was found that the first barrier to implement research utilization was an organizational factors such as: lack of time providing by the organization to nurses in order to read research, the shortage of staff nurses hinders the implementation of new evidences, lack of availability of resources (e.g. equipment) or new instruments in the clinical areas that needed to implement some research findings, and lack of enough authority to change patient care procedures according to research findings. The second ranked barrier was the lack of consistency between education and practice in nursing, and the lack of organizational and administrative motivation for the employee to do research was ranked as the third barrier, this barrier could be explained by the lack of interest of administrators and decision makers to change [38].

Furthermore, the controversies and contradictions between published recommendations regarding nursing interventions of SSI prevention was the third main factor affect the knowledge scores; in this study, the last question of the questionnaire, which asked about the recommended time of pre-operative hair removal in elective surgery, thirty six percent $(n=72)$ of the study participants were answered it correctly as "immediately before surgery" and sixty four percent $(\mathrm{n}=128)$ did not [39] [40] found in their clinical trials that patients who 
shaved the site of surgery at the day before the surgery day developed SSIs less than those who shaved the site of surgery at the day of surgery. Moreover [26], found in their experimental study that using clipper on the night before an operation and a $50 \mathrm{ml}$ chlorhexidine bath excluding head area preoperatively are useful to reduce postoperative SSI. In contrast [2], reported that preoperative hair removal must be performed as close to the surgery time as possible. However [41], conducted a systematic review regarding preoperative hair removal including eleven randomized controlled trials and they concluded that there was insufficient evidence to state when is the best time to remove hair. Such contradictory findings may affect nurses' conception regarding the proper measures to be implemented to prevent SSIs and put them in state of uncertainty.

In contrast, the scores that shifted the data toward high level of knowledge could be attributed primarily to many other factors that include: the basic knowledge that nurses had gained in their nursing schools, personal working experience in nursing, information gained through discussion with other health care providers, and information from policy and procedures. This claim is congruent with the findings of a recent Jordanian study that was aimed to explore the sources of Jordanian registered nurses' knowledge that used during their practice, it was found that most frequently used sources of knowledge are: firstly, the information gained during nursing education and secondly, the information from policy and procedure manuals [42]. Moreover, it was concordant with a study conducted in 2015 which reported that the basic nurses' knowledge of infection and its control measures come from the general information gained in basic schools of nursing [36]. However, Nurses experience can positively impact their knowledge. This idea was emphasized by Patricia Benner in 1982, when she published her theory "novice to expert" which report that the experience in clinical practice can continuously expand nurses' knowledge base; she categorized nursing experience to five levels; begin from novice who is beginner with no experience, she described the aspects of this category by; uncertainty, lack of confidence and frequent asking. Followed by the advanced beginner who characterized by the need of constant supervision; then, the competent nurse who has gained two to three years of experience in the same work area or in similar day to day situations. Thereafter, the proficient nurse who become perceive and understand the situations as a whole parts and who learned from experiences what to expect in certain situations and how to modify plans. Finally, the expert nurse who has an intuitive grasp of every clinical situations, this grasp come from the exposure to different situations and challenges [43].

Moreover, the accessibility of the information that can be gained from policy and procedure manuals and the convincing of these information put it as one of the top five sources of knowledge and let the nurses depend on these information when they need to get a fast answers for their questions in certain situations [44].

Spearman's correlations analysis were indicated that there were statistically significant relationships between all selected personal characteristics measured 
at the continuous level: age, total years of work experience in nursing, total years of work experience in surgical care unit, number of credit hours spent in surgical training courses, and monthly income. The age, total years of work experience alongside income were significantly correlated at $p>0.01$, this correlation may come from a logical link between these continuous variables, where the increase in the total years of work experience is axiomatically linked with aging and increasing monthly income and vice versa. The working experience in nursing ranked as the second source of knowledge utilized in practice among Jordanian registered nurses [42]. Moreover, Benner's theory was confirmed this idea by adopting Dreyfus brothers model of skills acquisition. However, Benner's believes that the experience in clinical settings allows nurses to continuously expand their knowledge base, and provide holistic patient care [43]. On the other side, culturally, the aging people in Jordan are supposed to be more aware, responsible, and loyal to their work. This claim may attribute to cultural issues such as; the cultural image of the older persons that is expected from them the idealism and a good reputation in their work. Alongside to the awareness of the humanitarian nursing role regarding patients' quality of life and safety.

However, in a recent study which conducted to evaluate the effects of gender, age, and educational level on the level of emotional awareness, it was reported that emotional awareness depends on the cultural context and generational societal teachings [45].

The analysis of Mann-Whitney U-test showed that there is a significant difference in nurses' knowledge regarding evidence based guidelines for the prevention of SSIs between those who are attending to special surgical related training course and those who are not. Furthermore, there were no statistical significant differences detected in nurses' knowledge regarding evidence based guidelines for the prevention of SSIs with respect to gender, marital status, and level of education subgroups. However, the statistical significant difference in participants' knowledge regarding evidence based guidelines for the prevention of SSIs between those who are attending to special surgical related training course and those who are not attributed to; nurses' exposure to the new recommendations regarding evidence based guidelines for the prevention of SSIs, and retrieving their knowledge regarding evidence based guidelines for the prevention of SSIs. Moreover, the difference between married and unmarried participants was approaching significant $P$-value $=0.053$. Furthermore, this approaching difference can be referred to the variations between married and unmarried nurses which is attributed to; firstly, the sense of responsibility; the married persons in Jordanian culture have a bulk of responsibilities that may let them more loyal to their work and more constant. Secondly, age and goal orientation; in the last three decades the average marriage age in Jordan was raised because of the economic hardship that affect the country and other regional geopolitical events. In an article addressing changes in marriage trends in Jordan, it was report that $94 \%$ of men between ages 20 and 24 were single and $67 \%$ of women in the age of 20 - 24 were unmarried [46]. Furthermore, the older aged nurses are more aware and goal oriented compared to younger adults. However, it has been found that 
goal oriented persons have more occupational well-being and work engagement than others [47]. Thirdly, the number of years of work experience; nursing experience can develop nursing knowledge, and also, it can change nurses' way of thinking and their decision making regarding serious situations [43]. And finally, monthly income was increased by aging and years of experience. This factor can directly support the marriage persons psychosocially and enhance their satisfaction to be more loyal to their work and more constant. Generally, higher income is one of the most important factors that enhance job satisfaction among California registered nurses, which primarily attributed to the sense of secure life [48]. However, the overall difference in marital subgroups in this study was negligible or inconsiderable. Moreover, Labeau et al. (2010) found that there was no significant difference between marital status subgroups with respect to nurses' knowledge regarding evidence based guidelines for the prevention of SSIs. However, in this study there was no significant difference in gender and level of education with respect to the total knowledge scores. In comparison to other research findings, there were a significant difference in knowledge scores of gender subgroup, where the male nurses' knowledge regarding evidence based guidelines for the prevention of SSIs were significantly better than female nurses; however, there were no significant differences in knowledge scores between the other subgroups [16].

Stepwise regression analysis was employed to determine the most significant predictors from age, gender, marital status, total years of work experience in nursing, total years of work experience in surgical care unit, level of education, number of surgical courses' accredited hours, and monthly income in predicting total knowledge regarding evidence based guidelines for the prevention of SSIs. Results showed that the most significant predictors were only two variables; firstly, number of credit hours attended by nurses for surgical-related training courses which explained $10.9 \%$ of variance in nurses' total knowledge scores. In general, it's intuitive that continuing education and training courses have a crucial role in improving nurses' knowledge. Moreover, the number of credit hours attended by nurses for surgical-related training courses reflects the time spent in studying the topic and reviewing the updates regarding evidence based guidelines for the prevention of SSIs which intuitively predict some percent of total knowledge score. However, this claim was congruent with a study that was conducted to evaluate the impact of a cancer nursing education course on cancer nursing-related knowledge, it was found that nurses who attended the nursing education course extremely improved their cancer nursing-related knowledge compared to those who was not attended [49]. Secondly, adding the total years of work experience in nursing to the regression model explained $5.8 \%$ of variance in nurses' total knowledge scores. The work experience considered as a source of knowledge as claimed by [25].

\section{Study Limitations}

There are several limitations in this study that need to be taken into consideration. Firstly, the major limitation of the current study was related to the main 
instrument used, although it is a valid measure to assess nurses' knowledge regarding evidence based guidelines for the prevention of SSIs in Western culture, this instrument may lack the sensitivity to measure the same construct in the Jordanian culture. In addition, guidelines can change over time, thus, adaptation and re-evaluation of the instrument will be needed every time when a newly published evidence for the prevention of SSI is cited. Secondly, the use of a convenience sampling from governmental and university-affiliated sectors, and did not include nurses from military or private sector, therefore, limit the external validity (generalizability) of the results and create selection bias of the sample. Thirdly, the use of cross-sectional design considered as a weakness of this study where more controlled research design could have been employed to control for confounding variables. However, according to our knowledge, this is the first study of its kind assessing Jordanian nurses' knowledge regarding evidence based guidelines for the prevention of SSIs conducted at the national level.

\section{Conclusion}

The results of this study shed light on the obstacles that hamper the Jordanian nurses' knowledge regarding evidence based guidelines for the prevention of SSIs in acute care settings. However, the successful implementation of infection control measures, particularly SSI prevention measures, and well-structured continuing education programs are considered as a substantial element that would improve nurses' knowledge regarding evidence based guidelines for the prevention of SSIs and ultimately leads to positive impacts on surgical patients admitted to the acute care settings in terms of patients' quality of care, and their safety. Thus, hospital administrators and all other parties involved should continue to emphasize more on the educational needs of general nurses population, particularly those working in acute care settings in order not to compensate quality of care delivered in acute care settings. Researchers in nursing discipline must also continue exploring the factors that may significantly affect nurses' knowledge regarding evidence based guidelines for the prevention of SSIs employing well-controlled research design.

\section{References}

[1] CDC (2012) Surgical Site Infection (SSI). Centers for Disease Control and Prevention, Atlanta.

[2] Harrington, P. (2014) Prevention of Surgical Site Infection. Nursing Standard, 28, 50-58. https://doi.org/10.7748/ns.28.48.50.e8958

[3] NICE (2008) Surgical Site Infection: Prevention and Treatment of Surgical Site Infection. Clinical guideline [CG74], National Institute for Health and Care Excellence, London.

[4] Nwankwo, E.O., Ibeh, I. and Enabulele, O.I. (2012) Incidence and Risk Factors of Surgical Site Infection in a Tertiary Health Institution in Kano, Northwestern Nigeria. International Journal of Infection Control, 8, 1-6. https://doi.org/10.3396/ijic.v8i4.035.12

[5] Patel, S.M., et al. (2012) Surgical Site Infections: Incidence and Risk Factors in a 
Tertiary Care Hospital, Western India. National Journal of Community Medicine, 3, 193-196.

[6] Kosuke, I., et al. (2014) Incisional Surgical Site Infection after Elective Open Surgery for Colorectal Cancer. International Journal of Surgical Oncology, 2014, Article ID: 419712, 5 p.

[7] Al-Zaru, I.M., AbuAlRub, R. and Musallam, E.A. (2011) Economical and Clinical Impact of Surgical Site Infection Following Coronary Artery Bypass Graft Surgery in North Jordan. International Journal of Nursing Practice, 17, 117-125. https://doi.org/10.1111/j.1440-172X.2011.01916.x

[8] Gould, D. (2012) Causes, Prevention and Management of Surgical Site Infection. Nursing Standard, 26, 47-56. https://doi.org/10.7748/ns.26.47.47.s51

[9] Teija-Kaisa, A., et al. (2013) Risk Factors for Surgical Site Infection in Breast Surgery. Journal of Clinical Nursing, 22, 948-957. https://doi.org/10.1111/jocn.12009

[10] Shaffer, V.O., et al. (2014) Improving Quality of Surgical Care and Outcomes: Factors Impacting Surgical Site Infection after Colorectal Resection. American Surgeon, 80, 759-763.

[11] Quinn, A., Hill, A.D.K. and Humphreys, H. (2009) Evolving Issues in the Prevention of Surgical Site Infections. The Surgeon: Journal of the Royal Colleges of Surgeons of Edinburgh and Ireland, 7, 170-172. https://doi.org/10.1016/S1479-666X(09)80041-3

[12] Tsai, D.M. and Caterson, E.J. (2014) Current Preventive Measures for Health-Care Associated Surgical Site Infections: A Review. Patient Safety in Surgery, 8, 1-25. https://doi.org/10.1186/s13037-014-0042-5

[13] Alfonso, J.L., et al. (2007) Are We Really Seeing the Total Costs of Surgical Site Infections? A Spanish Study. Wound Repair \& Regeneration, 15, 474-481. https://doi.org/10.1111/j.1524-475X.2007.00254.x

[14] Rusu, D., Rusu, G.G. and Bulicrea, R. (2013) Surgical Site Infections: Incidence and Additional Costs. Public Health and Management, II, 227-230.

[15] Mangram, A.J., et al. (1999) Guideline for Prevention of Surgical Site Infection, 1999. Centers for Disease Control and Prevention (CDC) Hospital Infection Control Practices Advisory Committee. American Journal of Infection Control, 27, 97 134. https://doi.org/10.1016/S0196-6553(99)70088-X

[16] Labeau, S.O., et al. (2010) Nurses' Knowledge of Evidence-Based Guidelines for the Prevention of Surgical Site Infection. Worldviews on Evidence-Based Nursing, 7, 16-24. https://doi.org/10.1111/j.1741-6787.2009.00177.x

[17] Farrelly, R. (2014) NHS Nurses' Fight against Infection. British Journal of Nursing, 23, 121. https://doi.org/10.12968/bjon.2014.23.2.121

[18] Zarchi, K., et al. (2014) Significant Differences in Nurses' Knowledge of Basic Wound Management-Implications for Treatment. Acta Dermato-Venereologica, 94, 403-407. https://doi.org/10.2340/00015555-1770

[19] Safdar, N. and Abad, C. (2008) Educational Interventions for Prevention of Healthcare-Associated Infection: A Systematic Review. Critical Care Medicine, 36, 933 940. https://doi.org/10.1097/CCM.0B013E318165FAF3

[20] Belowska, J., Panczyk, M. and Gotlib, J. (2014) Comparison of Knowledge and Attitudes towards the Use of Scientific Research in Clinical Practice among Departmental and Charge Nurses. Polish Journal of Public Health, 124, 138-144. https://doi.org/10.2478/pjph-2014-0031

[21] Scott, K., et al. (2012) Knowledge and Skills of Cancer Clinical Trials Nurses in Australia. Journal of Advanced Nursing, 68, 1111-1121. 
https://doi.org/10.1111/j.1365-2648.2011.05816.x

[22] PHE (2014) Surveillance of Surgical Site Infections in NHS Hospitals in England 2013/14. Public Health England, London.

[23] Cutting, K.F. and White, R. (2004) Defined and Refined: Criteria for Identifying Wound Infection Revisited. British Journal of Community Nursing, 9, S6-S15. https://doi.org/10.12968/bjcn.2004.9.sup1.12495

[24] Jidéus, L., Liss, A. and Ståhle, E. (2009) Patients with Sternal Wound Infection after Cardiac Surgery Do Not Improve Their Quality of Life. Scandinavian Cardiovascular Journal, 43, 194-200. https://doi.org/10.1080/14017430802573098

[25] Cahill, J.L., et al. (2008) Quality of Life after Infection in Total Joint Replacement. Journal of Orthopaedic Surgery (Hong Kong), 16, 58-65. https://doi.org/10.1177/230949900801600115

[26] Dizer, B., et al. (2009) The Effect of Nurse-Performed Preoperative Skin Preparation on Postoperative Surgical Site Infections in Abdominal Surgery. Journal of Clinical Nursing, 18, 3325-3332. https://doi.org/10.1111/j.1365-2702.2009.02885.x

[27] Al Maqbali, M.A. (2013) Preoperative Antiseptic Skin Preparations and Reducing SSI. British Journal of Nursing, 22, 1227-1233. https://doi.org/10.12968/bjon.2013.22.21.1227

[28] Beldi, G., et al. (2009) Impact of Intraoperative Behavior on Surgical Site Infections. The American Journal of Surgery, 198, 157-162. https://doi.org/10.1016/j.amjsurg.2008.09.023

[29] Cabaluna, N.D., et al. (2013) A Randomized, Double-Blinded Placebo-Controlled Clinical Trial of the Routine Use of Preoperative Antibiotic Prophylaxis in Modified Radical Mastectomy. World Journal of Surgery, 37, 59-66. https://doi.org/10.1007/s00268-012-1816-5

[30] Yan, R.C., et al. (2011) The Role of Prophylactic Antibiotics in Laparoscopic Cholecystectomy in Preventing Postoperative Infection: A Meta-Analysis. Journal of Laparoendoscopic \& Advanced Surgical Techniques, 21, 301-306. https://doi.org/10.1089/lap.2010.0436

[31] Wanzer, L., Goeckner, B. and Hicks, R.W. (2011) Perioperative Pharmacology: Antibiotic Administration. Third in a Series. AORN Journal, 93, 340-351. https://doi.org/10.1016/j.aorn.2010.08.026

[32] Downie, F., et al. (2010) Barrier Dressings in Surgical Site Infection Prevention Strategies. British Journal of Nursing, 19, S42-6. https://doi.org/10.12968/bjon.2010.19.Sup10.79693

[33] Labrague, L.J., et al. (2012) Operating Room Nurses' Knowledge and Practice of Sterile Technique. Journal of Nursing \& Care, 1, 113. https://doi.org/10.4172/2167-1168.1000113

[34] Jain, M., et al. (2012) Infection Control Practices among Doctors and Nurses in a Tertiary Care Hospital. Annals of Tropical Medicine and Public Health, 5, 29-33. https://doi.org/10.4103/1755-6783.92877

[35] Cicolini, G., et al. (2014) Nurses' Knowledge of Evidence-Based Guidelines on the Prevention of Peripheral Venous Catheter-Related Infections: A Multicentre Survey. Journal of Clinical Nursing, 23, 2578-2588. https://doi.org/10.1111/jocn.12474

[36] Teshager, F.A., Engeda, E.H. and Worku, W.Z. (2015) Knowledge, Practice, and Associated Factors towards Prevention of Surgical Site Infection among Nurses Working in Amhara Regional State Referral Hospitals, Northwest Ethiopia. Surgery Research and Practice, 2015, Article ID: 736175, 6 p.

[37] Momani, M. (1999) Assessment of Nurses' Continuing Education Needs in Jordan. 
The Journal of Continuing Education in Nursing, 30, 176-181.

[38] Al-Ghabeesh, S., Abu-Moghli, F. and Suleiman, K. (2014) Predictors of Research Utilization among Jordanian Registered Nurses: A Descriptive Correlational Study. International Journal of Medicine and Medical Sciences, 47, 1.

[39] Alexander, J.W., et al. (1983) The Influence of Hair-Removal Methods on Wound Infections. Archives of Surgery, 118, 347-52. https://doi.org/10.1001/archsurg.1983.01390030079013

[40] Pre-Operative Hair Removal to Reduce Surgical Site Infection (2008). Australian Nursing Journal, 15, 27-29.

[41] Tanner, J., Moncaster, K. and Woodings, D. (2007) Preoperative Hair Removal: A Systematic Review. Journal of Perioperative Practice, 17, 118.

[42] Al-Ghabeesh, S.H., et al. (2013) Exploring Sources of Knowledge Utilized in Practice among Jordanian Registered Nurses. Journal of Evaluation in Clinical Practice, 19, 889-894.

[43] Benner, P. (1982) From Novice to Expert. American Journal of Nursing, 82, 402 407. https://doi.org/10.1097/00000446-198282030-00004

[44] Oh, E.G. (2008) Research Activities and Perceptions of Barriers to Research Utilization among Critical Care Nurses in Korea. Intensive and Critical Care Nursing, 24, 314-322. https://doi.org/10.1016/j.iccn.2007.12.001

[45] Nandrino, J.-L., Baracca, M., Antoine, P., Paget, V., Bydlowski, S. and Carton, S. (2013) Level of Emotional Awareness in the General French Population: Effects of Gender, Age, and Education Level. International Journal of Psychology, 48, 1072-1079. https://doi.org/10.1080/00207594.2012.753149

[46] Peskin, D. (2010) Economic Hardship Changes Marriage Trends in Jordan. United Nations Children's Fund, 2, 12.

[47] Adriaenssens, J., De Gucht, V. and Maes, S. (2015) Association of Goal Orientation with Work Engagement and Burnout in Emergency Nurses. Journal of Occupational Health, 57, 151-160. https://doi.org/10.1539/joh.14-0069-OA

[48] Tellez, M. (2012) Work Satisfaction among California Registered Nurses: A Longitudinal Comparative Analysis. Nursing Economic\$, 30, 73-81.

[49] Steginga, S.K., et al. (2005) Impact of an Intensive Nursing Education Course on Nurses' Knowledge, Confidence, Attitudes, and Perceived Skills in the Care of Patients with Cancer. Oncology Nursing Forum, 32, 375-381.

Submit or recommend next manuscript to SCIRP and we will provide best service for you:

Accepting pre-submission inquiries through Email, Facebook, LinkedIn, Twitter, etc. A wide selection of journals (inclusive of 9 subjects, more than 200 journals)

Providing 24-hour high-quality service

User-friendly online submission system

Fair and swift peer-review system

Efficient typesetting and proofreading procedure

Display of the result of downloads and visits, as well as the number of cited articles

Maximum dissemination of your research work

Submit your manuscript at: http://papersubmission.scirp.org/

Or contact ojn@scirp.org 